teeth, and construction of partial dentures.

If dental radiographs are procurable, and it is the policy to have all recruits so radiographed, the dental oral surgeon or dentist will see that all foci of infection have been eradicated, because an injury or wound which lowers the resistance of the part, invites metastatic complications from these foci and greatly endangers the patient.

The dental oral surgeon will be called upon to assist the medical oral surgeon or operating surgeon, as the case may be. This assistance may be in plastic surgery and bone work, making of splints and mechanical appliances designed to maintain the occlusion of remaining teeth until final restoration may be attempted, this implies recovery from infection.
So far as possible the dental oral surgeon should familiarize himself with the treatment of wounds both sterile and infected, control of hemorrhage by packing or ligation of arteries, in continuity, he should anticipate secondary hemorrhages in cases of infection. Above all he should develop an aseptic technic, he should have a practical knowledge of anesthetics, both general and local, including intra-trachial, or thru a tracheotomy tube and pharyngeal.

To sum it all up he should improve every opportunity offered by a surgical clinic and apply his dental knowledge to cases which involve the teeth, jaws or soft part about the face and neck.

I do not doubt that plenty of opportunities will be offered to the man to do all that he is prepared and able to do even tho he is not a ranking officer.

\title{
AN IMPROVED METHOD OF STERILIZATION OF INFECTED TEETH.
}

By Montgomery LaRoche, D.D. S., New York City.

$\mathrm{B}$ EING confronted, as most of us are, with the problem of sterilization of devitalized and infected teeth and roots, I have often been baffled in my efforts in this direction but after trying many different procedures often with unsatisfactory results, I have developed a technic which I have found to be efficacious in all of the many tests to which it has been subjected for some time past. In the course of my endeavors in this direction, I have tried first one thing and then another, and found that each and every method had one or more flaws in it somewhere and therefore had to be abandoned as unsatisfactory. With the increased importance of the subject as shown by the investigations of the past few years, in regard to the relation of infection to systemic disease, I redoubled my efforts to obtain the ideal which has been set before us and which I was endeavoring to reach.

We are all no doubt quite familiar with Rhein's technic. He and Callahan as well as Prinz must be thoroly studied by students who would get to the heart of this matter. In my experience I have been saved many wanderings, and in fact have been put upon what I am con- 
vinced to be right track by these great leaders of thought in the dental world.

In the light of recent knowledge on this subject our procedures are reduced to two, as it appears to me. We have drugs and chemicals on one hand and drugs and chemicals plus the electric current on the other. There is still some little dispute as regards the value of the galvanic current as used in ionization, but I feel confident that there is no longer room for doubting its efficacy, as I hope to show in this paper.

We must keep two objects clearly in view: The first, and I consider the most important upon the technical side, is the complete removal of the infection. The second is the relief and prevention of pain. If we do these things and do them well, it has yet to be proven that we should not save all such teeth for many years of continued health and usefulness.

- The infection to be removed must be considered as not only being in the tooth root itself but also in the surrounding area of granulation tissue and bone at and beyond the apex, which area may or may not show in the radiograph. The use of Sulphuric Acid or Sodium-Potassium or Tri-creosol-formalin or Howe's ammonium-silver nitrate and formalin solutions will undoubtedly sterilize the infected dentin, at least on the surface. But to attempt to sterilize the surrounding bone areas with any or all of these, and these alone, is to invite all kinds of disasters, including excessive and unnecessary pain and discomfort to the patient and failure in obtaining the object, among the most important. The same objections apply to the solutions generally used in ionization, viz: zinc or sodium chloride.

Of course it is understood that the rubber dam must be applied and the most rigid and exacting technic be regarded thruout the entire operation whenever the treatment of a root is under consideration. We as dentists can not be too exacting in this regard, and with a little practice it will be found to become second nature.

In opening a root $I$ find it to be very helpful to sterilize the pulp chamber and orifices of the roots with a solution of silver nitrate ten to twenty per cent. The debris should be removed carefully and thoroly and a clear way opened to and thru the root apex. Sodium-potassium and Sulphuric Acid are best used for this purpose, together with the proper instruments for the case in hand. The best practice is not to use engine drills, hand instruments being far more satisfactory. If engine drills are used at all great carc must be used to avoid making a step in the canal wall or drilling thru the side of the root.

Having arrived thus far we now have the problem of complete sterilization of the remote areas to contend with. We must consider under this head the dicep layers of dentin as well as the apical bone areas. The destruction of the micro-organisms in these areas presents the most difficult problem we have to contend with in this connection. But unless we can solve it, we have no right to allow these teeth to remain in the jaws of our patients.

We must consider that we have now arrived at the stage where we have a perfectly clean and open canal with passage thru the apex. The next step is to inject into the canal and thru the apex, using very little force and flooding the pulp chamber, a solution of beechwood creosote, boracic acid, alcohol and water, with a sterile syringe. I use for this purpose an ordinary "Sub-Q" dental syringe, which has been freshly steriled. The solution is made by mixing $95 \%$ alcohol and distilled water half and half and adding creosote and boracic acid to saturation. The platinum-iridium electrode is then passed into the canal, a sterile paper point passed down along side it as far as it will go and the excess packed around the electrode, the excess 
moisture carefully removed from the surrounding area and care being taken to avoid the possibility of a short circuit.

The next step must be taken with great care. If, after proper bacteriological investigation the root is found to be infected, the negative pole must be used in the tooth for the first treatment and possibly the second. The amount of current to be used must be determined by the sensations of the patient. My practice is to run the current up to where a distinct sensation is felt and then immediately drop it back to where practically none is felt, reducing it in the majority of instances about $0.2 \mathrm{M}$. A. I attempt to maintain this strength of current for 15 minutes. However, this is not always possible, due to the drying out of the root, which increases the resistance, and the occasional increase in the sensitiveness of the surrounding area. Under no circumstances is it necessary for the patient to receive a distinct pain and when this occurs the current must be reduced or discontinued.

On the third treatment the positive pole is used in the tooth, using the same solution in the same way and after the fourth treatment it is usually advisable to take a culture, using the method as described in my paper which will appear in the Journal of the Allied Dental Societies for June, 1918. Too much stress cannot be laid on the checking up of the result of our sterilization bacteriologically.

If after proper incubation the culture shows the area be still infected a continuance of the treatment must be made, but if the canal is thoroly cleansed and the apex open, four treatments as described will usually suffice.

In explanation I would say that in using the negative pole first, we avoid spreading the bacteria and their products thru the tissues by the cataphoresis set up by the current. Extreme neuralgic conditions have been caused by this occurrence, both in my own experience and in that of others. In fact I have brought about the relief of this condition caused by using first the positive pole in the tooth. And I have yet to experience any such neuralgic condition irrought about by using the current in the directions described.

The solution is not only germicidal, but is healing and preservative. I have found that the zinc or sodium chloride solutions, used either with the zinc electrode or the platinum electrode, cause a certain amount of irritation at the apex, which is entirely lacking when the alcoholic solution of creosote and boracic acid described above is used. Furthermore you are in position to know to a certainty whether or not you bave sterilized the tissues involved.

To sum up we have the following salient points:

1. The sterilization of the infected structures.

2. The ability to prevent pain.

3. The ability to prevent the spread of the infection in the tissues thru the use of the cataphoric current infected areas.

4. No discoloration results, and

5. The simplification of our root canal technic. 